\title{
Secreted miRNAs in the tripartite neuromuscular junction
}

\author{
Liangsheng Lu', Yuan Liu', Yuyan Liu', Fan Zhang ${ }^{1}$, Han Wang ${ }^{1}$, Qipeng Zhang ${ }^{1 *}$ and Feng Pan²
}

\begin{abstract}
microRNAs are small, non-coding, single-stranded RNAs that can suppress mRNA translation at the posttranscriptional level by binding to imperfect complementary sequences on mRNA targets and then cause their degradation or hinder protein translation. Recently, bunch of evidence showed that microRNAs (miRNAs) existed in presynaptic and postsynaptic parts and implicated in the synapses formation and pruning during development and the modulation of synaptic plasticity in the adult stage. Besides working intracellularly, we previous reported that miRNAs also could be secreted and became extracellular miRNAs, these extracellular miRNAs could be uptake by postsynaptic-density enriched fragment and play important roles in synapse. As a special type of synapse, the neuromuscular junction (NMJ) has three different parts: the muscle fiber, the motor neuron axon terminal and the perisynaptic Schwann cells. There are microRNA targeting mRNAs in NMJ and the local translation of the mRNA which contribute to the NMJ formation, maintenance or re-innervation. Interestingly, we noted that a myo specific miRNA, miR-206 has the potential binding sites on neuronal expressing genes' $3^{\prime}$-untranslated region (3'-UTR). In this perspective review, we assayed the miR-206 and its targeting mRNA expression in muscles and neurons and analyzed the possibility of secreted miRNAs and their potential roles in NMJ.
\end{abstract}

Keywords: Mir-1/Mir-206, Skeletal muscles, Secretion, Local translation, Neuro-muscular junction

\section{Background}

\section{The history of myomiRs}

MicroRNAs are temporally expressed and play important roles in neuromuscular junction (NMJ) maturation, maintenance and injury-recovery process. In 2008, two groups both reported that let-7 influence the maturation of abdominal NMJs during metamorphosis [1,2]. Simon at al. showed that high conserved muscle specific miR-1 regulates the synaptic transmission at neuromuscular junctions by postsynaptic suppressing the nicotinic acetylcholine receptors expression and presynaptic regulating the transcription factor myocyte enhancer factor-2 [3]. Furthermore, some activity-regulated specific miRNAs as well as their targeted mRNA in NMJ are reported and indicating that miRNA pathway could coordinate the gene expression at NMJ in an activity-dependent manner [4].

\footnotetext{
* Correspondence: qpzhang@nju.edu.cn; fengpanmd@126.com

${ }^{1}$ State Key Laboratory of Pharmaceutical Biotechnology, Jiangsu Engineering Research Center for microRNA Biology and Biotechnology, School of Life Sciences, Nanjing University, Nanjing, China

${ }^{2}$ Department of Andrology, The Affiliated Obstetrics and Gynecology

Hospital of Nanjing Medical University, Nanjing Maternity and Child Health Care Hospital, Nanjing, China
}

In fact, there are some tissue-specific or tissue-enriched miRNAs which implied their remarkable roles in tissue specification or cell lineage decisions. Canonically, three "myomiRs" (miR-1, miR-133a and miR-206) are highly enriched in both heart and skeletal muscle [5]. myomiRs even are essential in muscle development, which is first shown by Sokol and Ambros, who reported that deletion of miR-1 in flies will lead to a premature death because of a failure of skeletal muscle to properly grow during the larva development stage [6].

Among the three myomiRs, miR-206 is a strictly skeletal-muscle specific microRNA and miR-206 also is one of the most abundant miRNAs expressed in skeletal muscle. Since 2006, bunch of results reveal the multiple function of miR-206 in vitro and in vivo: (1) miR-206 promoted muscle differentiation by inhibiting Pola1 expression [7]; (2) miR-1 and miR-206 inhibits connexin $43(\mathrm{Cx} 43)$ mRNA translation during perinatal muscle development [8]; (3) Amyotrophic lateral sclerosis (ALS) a neurodegenerative disease characterized by progressively loss of motor neurons. However, knocking out miR-206 in G93A-SOD1 ALS mouse model could 


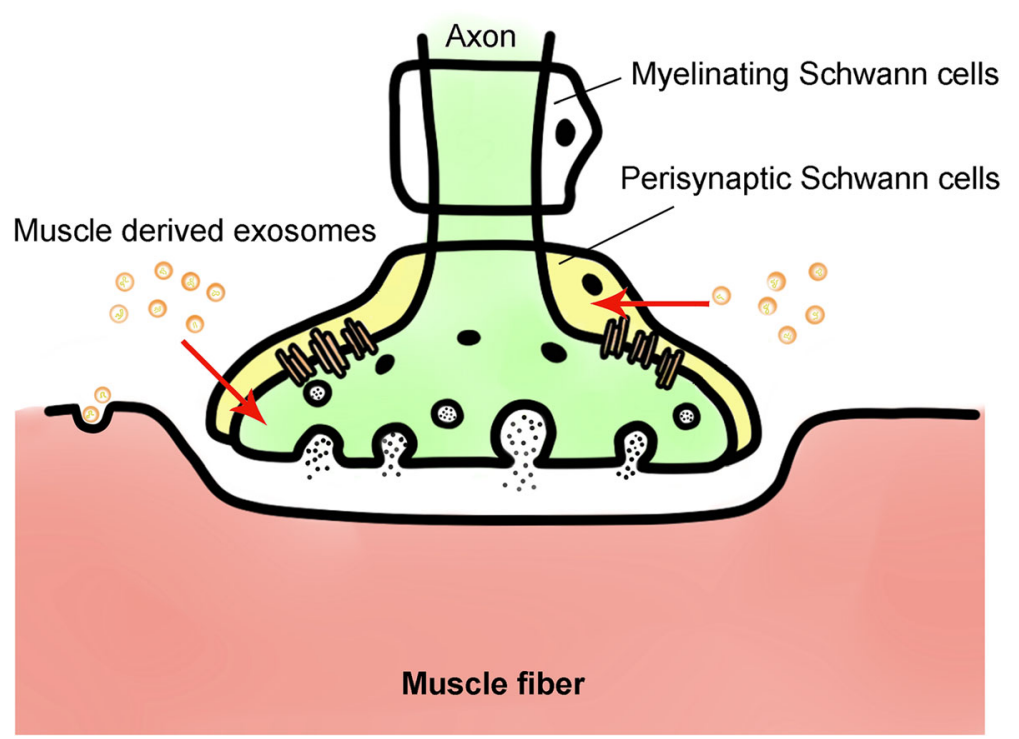

Fig. 1 Secreted microRNA in the tripartite neuromuscular junction. The neuromuscular junction has three different type cells: (1) muscle fiber and satellite cells; (2) axon terminals; (3) perisynaptic Schwann cells. The muscle specific miRNAs can be secreted via exosomes and diffuse to nerve terminal and/or perisynaptic Schwann cells where the exosomes are uptaken by these cells and play regulatory roles locally (arrow)

exacerbate the disease progression [9]. The authors showed that MiR-206 is required during the regeneration of neuromuscular synapses after surgical transection, and the miRNA-histone deacetylase 4 pathway contribute to the susceptibility to re-innervation of a muscle fiber and this theory answered that in ALS (motor neuron pathology plays a key role), whereas miR-206 is expressed exclusively in muscles, could counteract the pathology [10]. In fact, Velleca et al. found that the non-coding transcript $7 \mathrm{H} 4$ is synaptically enriched in 1994 [11]; and the 7H4 was later identified as the primary miRNA for miR-206 [12]. Interestingly, the $7 \mathrm{H} 4$ transcript, a muscle-specific and up-regulated upon denervation RNA are enriched in synaptic part.

\section{Main text}

Hypothesis: secreted microRNAs might be able to work in a trans-synapse way

In traditional model, the muscles fiber could release protein factor or nitric oxide neuromodulator to reversely influence their innervating axonal terminals [13]. Although there is no direct precise evidence of miR-206 location information in NMJ, we highly suspect that miR-206 might be trans-synaptic transported. we proposed that the miRNA affects nerve-muscle interactions via miRNA secretion between NMJ parts. There are some facts that consistent with our hypothesis.

As well known, both dendrites and axonal terminals own many mRNA and local protein synthesis. The local mRNAs translation occurring in axon or dendrites can provide a local and sustainable source of proteins at sites that far away from the neuronal cell body. Importantly, the local RNA content in synaptic parts varies at different status. Many mRNA transcripts are known to reside in developing axons, at beginning, the growth cones contain primarily mRNAs encoding translation machinery and cytoskeleton elements. While the local mRNA of the growth cones switch to a more complex set of genes encoding synaptogenesis-related proteins. Usually, Localized protein synthesis is regulated by neuronal activities, this mechanism provides an activity-dependent manner of supply novel proteins "at demands" [14, 15].

We take brain-derived neurotrophic factor (BDNF) as an example. BDNF is a neurotrophic factor for neuron's survival, growth and differentiation. Previous studies show that BDNF protects motor neurons from death and enhance their neurites growth, meanwhile BDNF also significantly inhibits the synaptogenesis. Furthermore, Song et al. recently reported that BDNF treatment on nerve-muscles co-culture in vitro could suppress NMJ formation and maturation and the authors further proved that this effect of BDNF is through a cAMP-PKA signaling pathway [16]. From this point, BDNF is inhibitory for NMJ re-innervation. It is reported that miR-206 repressed BDNF during myogenic differentiation in vitro, which promote us to suppose that whether miR-206 are secreted from muscles fiber (where abundantly miR-206 is expressed) and uptaken by axonal terminal (little miR-206 and many BDNF mRNA) and retrograde transport to motor neurons where it might suppress BDNF synthesis. 
Islamov et al. have shown that some coding mRNAs of synaptic enriched protein are existed at motor neuron axon terminals, for example, the synaptosome-associated protein of $25 \mathrm{kD}$, (SNAP25) mRNA are locally translated at motor neuron axon terminal. They further showed that If inject siRNAs, the protein synthesis is disturbed, which also prove that there is intense mRNA translation at presynaptic part [17]. We searched the possible regulatory miRNA of SNAP25 mRNA, a conserved binding site of miR-1/miR-206 is located at SNAP25 mRNA's 3'-UTR. Similarly, vesicle-associated membrane protein 2 (VAMP2), another main component of a protein complex locating at the presynaptic membrane, also have miR-1/miR-206 binding site at its 3'-UTR [18]. Those myomiR's binding site locating on the presynaptic specific gene indicate presynaptic might be one of the destinations of secreted muscle-specific miRNAs. Form this point, proper innervation is an essential condition for muscles healthy. As a far ending of neurons, the axon owns its local mRNA translation to keep its activity. Interestingly, the 3'-UTR of neuronal mRNAs have muscle specific miRNAs binding sites. This key point implied that the muscle-specific miRNAs might work in a trans-synaptic way, either retrograde transported to cell body or direct play role locally at presynaptic part.

How myomiRs was secreted from muscles fiber? Exosome, might be a possible answer. Exosomes are small vesicles released by almost all eukaryotic cells; proteins, mRNA and miRNA can be transferred to recipient cells and regulate cellular processes. In 2017, Gasperi et al. reported that skeletal muscle fibers release exosomes. Furthermore, Denervation resulted in a marked increase in miR-206 and reduced expression of miR-1, miR-133 in myofiber-derived exosomes [19]. These findings demonstrate that muscle cells release exosomes which can transfer biologically active miRNAs to recipient cells. However, whether recipient cells include axonal terminal is still not known. In fact, many studies show that the myomiRs (miR-1, miR-133, and miR-206) in serum are highly elevated in the serum of patients with Duchenne muscular dystrophy and in dystrophin-deficient animal models [20], which means that there must be lots of myomiRs are secreted, as a closer site, axon terminal should have a big chance to encounter the secreted miRNAs.

It has been reported that in the muscles, skeletal muscle specific miR-206 is secreted from activated satellite cells and play important regulatory roles in fibrogenic cell after uptaken by these cells [21]. As NMJ is the closest site where a neuron is contact with muscles, it is possible that muscles secreted miRNAs enter the axon terminals and regulate the local translation in the NMJ. With the advance of in situ hybridization technique, simultaneously detect microRNA and mRNA at NMJ should could provide lots of new information about the distribution of miRNA and mRNAs at NMJ.

Who are possible recipient cells: Besides axon, there is another candidate-perisynaptic Schwann cells (PSC).

Besides axon terminal and muscle fiber, NMJ also has a third type cells: perisynaptic Schwann cells (PSC), which developed from neural crest cells. Therefore, this is a tripartite neuromuscular junction. Recently, more and more research find that this PSC have dramatic influence on NMJ. First, although PSC are not essential to axonal growth and synapse formation, they help with the maintenance of NMJs, at least in frog [22]; second, besides a supportive role, the PSC also response to NMJ neurotransmitter transmission and even actively modulate the NMJ transmission [23, 24]; third, PSCs have important roles in the regeneration of nerve axons after nerve injury [25].

However, little is known about PSC biology. Whether PSC could produce and secret BDNF? whether PSC could uptake exosomes? If secreted mir-206 could enter PSC, whether the BDNF mRNA translation in PSC is suppressed. With high-resolution in situ hybridization methods, the information about mRNAs and miRNAs expression in axon terminal, muscle fiber and PSC could be analyzed.

\section{Conclusions}

As a classic model system of synapse, the NMJ has been studied for over a century. Much of our knowledge of synaptic structure, synapse physiology and synapse formation, maturation is obtained from NMJ. In many over-simplified synapse model, synapses only have two components: a presynaptic nerve terminal and a postsynaptic part, the neurotransmitter containing synaptic vesicles are stored at presynaptic site and the receptors are distributed on the postsynaptic density region [26]. However, in the updated NMJ anatomy, the neuro-muscle synapse is a tripartite neuromuscular junction. There are nerve terminals, glial cells and muscle fibers in this specific region. Therefore, the secretion and absorption of extracellular miRNAs is more complex: muscle specific miRNAs could be secreted and might be uptaken either by axon terminals or PSC. However, there is little study accessed this issue and further study is eagerly needed to provide the information about this tripartite neuromuscular junction (Fig. 1).

\footnotetext{
Abbreviations

3'-UTR: 3'-untranslated region; BDNF: brain-derived neurotrophic factor; MiRNA: microRNA; NMJ: neuromuscular junction; PSC: perisynaptic Schwann cells; SNAP25: synaptosome-associated protein of 25 kD; VAMP2: vesicleassociated membrane protein 2
} 


\section{Funding}

This work was supported by grants from the National Natural Science Foundation of China (31100777 and 81170309), the Fundamental Research Funds for the Central Universities (020814380018, 020814380077), The China Scholarship Council (CSC, No. 201606195024), and the Natural Science Foundation of Jiangsu Province (BK20160138).

\section{Availability of data and materials}

Not applicable.

\section{Authors' contributions}

$L S L, Y L, Y Y L$, and $F Z$ participated the discussion of manuscript, $H$ W draws the figures, QZ and FF wrote the manuscript. All authors read and approved the final manuscript.

\section{Ethics approval and consent to participate} Not applicable.

\section{Consent for publication}

Not applicable.

\section{Competing interests}

The authors declare no conflict of interest.

\section{Publisher's Note}

Springer Nature remains neutral with regard to jurisdictional claims in published maps and institutional affiliations.

Received: 4 July 2018 Accepted: 14 March 2019

Published online: 05 June 2019

\section{References}

1. Caygill EE, Johnston LA. Temporal regulation of metamorphic processes in Drosophila by the let-7 and miR-125 heterochronic microRNAs. Curr Biol. 2008;18(13):943-50.

2. Sokol NS, et al. Drosophila let-7 microRNA is required for remodeling of the neuromusculature during metamorphosis. Genes Dev. 2008;22(12):1591-6.

3. Simon DJ, et al. The microRNA miR-1 regulates a MEF-2-dependent retrograde signal at neuromuscular junctions. Cell. 2008;133(5):903-15.

4. Nesler KR, et al. The miRNA pathway controls rapid changes in activitydependent synaptic structure at the Drosophila melanogaster neuromuscular junction. PLoS One. 2013;8(7):e68385.

5. Sempere LF, et al. Expression profiling of mammalian microRNAs uncovers a subset of brain-expressed microRNAs with possible roles in murine and human neuronal differentiation. Genome Biol. 2004:5(3):R13.

6. Sokol NS, Ambros V. Mesodermally expressed Drosophila microRNA-1 is regulated by twist and is required in muscles during larval growth. Genes Dev. 2005;19(19):2343-54.

7. Kim HK, et al. Muscle-specific microRNA miR-206 promotes muscle differentiation. J Cell Biol. 2006;174(5):677-87.

8. Anderson C, Catoe H, Werner R. MIR-206 regulates connexin43 expression during skeletal muscle development. Nucleic Acids Res. 2006;34(20):586371.

9. Williams AH, et al. MicroRNA-206 delays ALS progression and promotes regeneration of neuromuscular synapses in mice. Science. 2009;326(5959): 1549-54.

10. Brown RH. Medicine. A reinnervating microRNA. Science. 2009;326(5959): 1494-5

11. Velleca MA, Wallace MC, Merlie JP. A novel synapse-associated noncoding RNA. Mol Cell Biol. 1994;14(11):7095-104.

12. Rodriguez $A$, et al. Identification of mammalian microRNA host genes and transcription units. Genome Res. 2004;14(10A):1902-10.

13. Zhu H, et al. Skeletal muscle calpain acts through nitric oxide and neural miRNAs to regulate acetylcholine release in motor nerve terminals. J Neurosci. 2013;33(17):7308-24.

14. Glock C, Heumuller M, Schuman EM. mRNA transport \& local translation in neurons. Curr Opin Neurobiol. 2017:45:169-77.

15. Costa CJ, Willis DE. To the end of the line: axonal mRNA transport and local translation in health and neurodegenerative disease. Dev Neurobiol. 2018; 78(3):209-20.
16. Song W, Jin XA. Brain-derived neurotrophic factor inhibits neuromuscular junction maturation in a CAMP-PKA-dependent way. Neurosci Lett. 2015; 591:8-12.

17. Islamov RR, et al. Synaptosome-associated protein 25 (SNAP25) synthesis in terminal buttons of mouse motor neuron. Dokl Biochem Biophys. 2015;464: 272-4.

18. Agarwal $V$, et al. Predicting effective microRNA target sites in mammalian mRNAs. Elife. 2015;4.

19. De Gasperi R, et al. Denervation-related alterations and biological activity of miRNAs contained in exosomes released by skeletal muscle fibers. Sci Rep. 2017;7(1):12888.

20. Coenen-Stass AML, Wood MJA, Roberts TC. Biomarker potential of extracellular miRNAs in Duchenne muscular dystrophy. Trends Mol Med. 2017;23(11):989-1001.

21. Fry CS, et al. Myogenic progenitor cells control extracellular matrix production by fibroblasts during skeletal muscle hypertrophy. Cell Stem Cell. 2017;20(1):56-69.

22. Armati, P.J., The biology of Schwann cells : development, differentiation and immunomodulation. 2007, Cambridge, UK ; New York: Cambridge University Press. xiii, 249 p., 16 p. of plates.

23. Belair, E.L., J. Vallee, and R. Robitaille, In vivo long-term synaptic plasticity of glial cells. J Physiol, 2010. 588(Pt 7): p. 1039-1056.

24. Darabid H, Arbour D, Robitaille R. Glial cells decipher synaptic competition at the mammalian neuromuscular junction. J Neurosci. 2013:33(4):1297-313.

25. Rupp A, et al. Motor nerve terminal destruction and regeneration following anti-ganglioside antibody and complement-mediated injury: an in and ex vivo imaging study in the mouse. Exp Neurol. 2012;233(2):836-48.

26. Balice-Gordon RJ. Dynamic roles at the neuromuscular junction. Schwann cells. Curr Biol. 1996;6(9):1054-6.

\section{Ready to submit your research? Choose BMC and benefit from:}

- fast, convenient online submission

- thorough peer review by experienced researchers in your field

- rapid publication on acceptance

- support for research data, including large and complex data types

- gold Open Access which fosters wider collaboration and increased citations

- maximum visibility for your research: over $100 \mathrm{M}$ website views per year

At BMC, research is always in progress.

Learn more biomedcentral.com/submissions 